\title{
A double blind placebo controlled study to determine the effects of intermittent cyclical etidronate on bone mineral density in patients on long term oral corticosteroid treatment
}

\author{
P Pitt, F Li, P Todd, D Webber, S Pack, C Moniz
}

\begin{abstract}
Background-A double blind, placebo controlled study was undertaken to determine the effects of 104 weeks of intermittent cyclical etidronate therapy on bone mineral density (BMD) in patients undergoing long term oral corticosteroid therapy.

Methods-Forty nine patients of mean age 59 years on long term (>6 months) corticosteroid treatment were randomised to receive either $400 \mathrm{mg} /$ day etidronate or placebo for 14 days followed in both groups by calcium (equivalent to $97 \mathrm{mg}$ elemental Ca/day) with vitamin D (400 IU) for 76 days. The cycle was repeated a total of eight times over a period of two years. Dual energy $x$ ray absorptiometry (DEXA) measurements of the lumbar spine and hip BMD and biochemical bone marker analyses were performed at baseline and every six months.
\end{abstract}

Results-Twenty six patients (10 men) received cyclical etidronate and 23 (nine men) received placebo. The mean (SD) dose of corticosteroid (prednisone or equivalent) at baseline in the etidronate group was 8 (4) $\mathrm{mg} / \mathrm{day}$ and in the placebo group was 7 (4) mg/day. Most of the patients $(43 \%)$ suffered from asthma. Forty one patients completed the study ( 22 in the etidronate group and 19 in the placebo group). All had a low BMD at entry and with treatment a significant difference was observed between groups in the mean (SE) percentage change from baseline in lumbar spine BMD at week 104 of $4.5(1.65) \%(p=0.007)$ with a $95 \%$ confidence interval (CI) of 1.12 to $7.87 \%$. No clinically or statistically significant treatment differences were observed at the hip or with bone markers. The incidence of adverse events was similar in the two groups.

Conclusions-The results show that intermittent cyclical etidronate therapy with calcium and vitamin $D$ supplementation significantly increases lumbar spine BMD in patients with osteoporosis resulting from long term treatment with corticosteroids.

(Thorax 1998;53:351-356)

Keywords: osteoporosis; corticosteroids; etidronate
It is well established that high dose corticosteroids can result in marked bone loss, especially during the early part of treatment. The exact dose at which this occurs is yet to be clearly defined although the general consensus indicates that $7.5-10 \mathrm{mg} /$ day of prednisone or its equivalent is associated with bone loss. These doses of corticosteroid are frequently indicated in patients with a wide range of chronic inflammatory, autoimmune and hypersensitivity conditions such as asthma, rheumatoid arthritis, polymyalgia rheumatica, and systemic lupus erythematosus. It is not uncommon for these patients to be treated with high $(>10 \mathrm{mg} /$ day $)$ doses for the first year and then a lower dose subsequently. The available evidence suggests that bone loss, although reversible, is greatest during the first six months ${ }^{1}$ and that up to $50 \%$ of patients taking corticosteroids long term will develop osteoporosis and experience vertebral crush fractures due to the associated decrease in bone mass. ${ }^{2}$

The aetiology of corticosteriod induced bone loss may be complex but is thought to involve the direct inhibition of the bone forming osteoblast and also affect overall calcium balance. Reduced bone formation may be a consequence of a reduction of osteoblast differentiation and an inhibition of the synthesis of osteoid by these cells. ${ }^{3}$ Corticosteroid excess also decreases intestinal absorption of calcium and its reabsorption by the renal tubule. ${ }^{1}$ The resulting secondary hyperparathyroidism leads to increased osteoclast activity and increased bone resorption. Furthermore, corticosteroids have been shown to reduce the production of oestrogen and testosterone by both direct and indirect mechanisms. ${ }^{4}$

Although the effects of corticosteroids on bone are well established, there are relatively few data available from well controlled, randomised clinical trials in the treatment of steroid induced bone loss. Studies with a number of agents such as oestrogen, calcitonin, vitamin $\mathrm{D}$, calcium, and bisphosphonates have all proved to be effective to varying degrees in the prevention of corticosteriod induced bone loss. However, these studies were generally small or not placebo controlled. Sambrook et at $\bar{p}$ showed that calcitriol $(0.5-1.0 \mu \mathrm{g} /$ day $)$ prevented bone loss from the spine more effectively than calcium alone ( $1000 \mathrm{mg} /$ day). Reid et $a l^{6} \mathrm{dem}-$ onstrated that the bisphosphonate pamidronate given orally at $150 \mathrm{mg} /$ day increased bone density at the spine after one year compared with placebo. 
More recently a series of studies with cyclical etidronate showed increases in bone mineral density at the spine and femoral neck. In the largest of these ${ }^{7} 42$ patients with corticosteroid induced osteoporosis were selected who had been taking cyclical etidronate and calcium and 46 patients who were taking calcium supplements alone were followed as a comparator group. After two years of treatment patients receiving etidronate had a mean increase in lumbar spine bone mineral density of $5.6 \%$ compared with a mean decrease of $1.8 \%$ in the calcium group. These studies suggest that the bisphosphonates may be a useful intervention in this disease.

The purpose of this study was to determine if an intermittent regimen of cyclical etidronate and calcium with vitamin D supplementation could prevent further bone loss in patients already receiving corticosteroids.

\section{Methods}

This was a double blind, placebo controlled, parallel group, single centre study. One hundred and thirty six patients (48 men) being treated with high dose corticosteroids were screened for eligibility for the study. These patients suffered from a variety of disorders including polymyalgia rheumatica, asthma, and systemic connective tissue disorders. Caucasian ambulatory patients aged 30 or over were selected for the study. To be eligible the patients had to have a lumbar spine bone mineral density (mean of L2-L4 vertebrae) of one standard deviation or more below the age and sex matched mean for the normal population, as determined by dual energy $x$ ray absorptiometry (DEXA), and to be receiving corticosteroids at doses equivalent to $5-20 \mathrm{mg}$ prednisolone per day, or at a dose of $20 \mathrm{mg}$ or more on an alternate day schedule. Patients had to have been receiving corticosteroids for at least six months prior to the start of the study and all asthmatics were receiving the maximum recommended doses of inhaled corticosteroids in addition to oral therapy. All patients indicated their willingness to participate by signing an informed consent form. Premenopausal women were excluded unless they had been hysterectomised or sterilised, as was any patient with generalised bone disease (including rheumatoid arthritis ${ }^{8}$ ) or those who had previously been treated with bone active agents in the therapeutic range or who had inflammatory bowel disease. ${ }^{9}$

Of the 136 patients screened, 49 were found to meet all of these criteria and were entered into the study. Patients were randomly assigned to an intermittent cyclical regimen of either etidronate ( $400 \mathrm{mg} /$ day) or placebo for 14 days followed in all patients by calcium (equivalent to $97 \mathrm{mg}$ elemental calcium/day) with vitamin D (400 units) for 76 days. The 90 day treatment cycle was repeated eight times over a period of 104 weeks. Administration of etidronate was oral, once daily, on an empty stomach-that is, two hours before or two hours after meals. Tablets were to be taken with water and not with milk or other dairy products. The calcium and vitamin $\mathrm{D}$ tablets were to be taken orally once daily, at the same time each day. Patients returned to the study centre at three month intervals.

\section{STUDY PROCEDURES}

Baseline assessments included physical examination, medical history, a determination of weekly calcium intakes, alcohol consumption and smoking habits, bone mass measurements, and routine clinical laboratory evaluations. Patients were then asked to return to the hospital at three monthly intervals. DEXA measurements of the lumbar spine and hip (femoral neck, trochanter, and Ward's triangle) were performed at the baseline visit and every six months thereafter using a Norland XR-26 Mark II bone densitometer with 2.2.5 series software and positioning aids. Operator precision was evaluated by repeated measurements performed on volunteers (departmental staff) 10 times over a two week period. Precision, expressed as a coefficient of variation, was lumbar spine $0.8 \%$, femoral neck $2.2 \%$, greater trochanter $3.4 \%$, and Ward's triangle $8.5 \%$. Stability of the densitometer was monitored through daily QC calibration and phantom measurements as per the manufacturer's instructions, and repeated phantom scans were performed at six monthly intervals using an Hologic spine phantom. The precision of these phantom scans over the duration of the study was $1.03 \%$ (expressed as a co-efficient of variation). DEXA measurements of 127 postmenopausal female volunteers were not significantly different from the normal database provided by the manufacturers. ${ }^{10}$ Clinical laboratory evaluations included serum and urine biochemistry analysis every six months and routine haematological analysis every 12 months during the study. Biochemical measurements relating to renal, bone and liver function were performed using standard chemistries on an automated analyser (Bayer, UK). Parathyroid hormone (Instar Ltd, Workingham, UK) and 25-hydroxy vitamin D (Nichols Institute Diagnostics Ltd, Newport, UK) were measured by immunoassay at baseline. Biochemical markers of bone turnover were measured every six months and included serum total alkaline phosphatase and urinary hydroxyproline/creatinine by colorimetric assay, and urinary pyridinoline/creatinine and urinary deoxypyridinoline/creatinine by HPLC techniques. ${ }^{11}$ Pyridinium compounds were performed by a single worker and, over the period of the study, within and between coefficients of variation were less than $10 \%$. Spinal radiographs were performed at baseline and every 12 months and were assessed at the end of the study in a blinded fashion by an independent radiologist using the method of Genant et al. ${ }^{12}$

The primary efficacy parameter was percentage change from baseline in bone mineral density (BMD) of the lumbar spine at week 104; the two treatment groups were compared using analysis of covariance (ANCOVA), adjusted for differences between groups in corticosteroid use or not. Mean daily corticosteroid dose during the study was the single covariate. The 
Table 1 Mean (SD) baseline demographic data

\begin{tabular}{lll}
\hline & \multicolumn{2}{l}{ Treatment group } \\
\cline { 2 - 3 } & Etidronate $(n=26)$ & Placebo $(n=23)$ \\
\hline Sex (men/women) & $10 / 16$ & $9 / 14$ \\
Age (years) & $58.9(13.7)(26)^{\mathrm{b}}$ & $59.2(10.8)(23)^{\mathrm{b}}$ \\
Years since natural menopause & $16.6(14.7)(11)$ & $15.7(7.9)(10)$ \\
Years since hysterectomy & $20.3(23.2)(3)$ & $10.9(8.6)(3)$ \\
Corticosteroid dose $(\mathrm{mg} / \mathrm{day})^{\mathrm{a}}$ & $8.2(4.2)(26)$ & $7.2(4.0)(23)$ \\
Lumbar spine BMD $\left(\mathrm{g} / \mathrm{cm}^{2}\right)$ & $0.74(0.12)(25)$ & $0.76(0.11)(21)$ \\
Lumbar spine T-score & $-2.50(0.77)(25)$ & $-2.42(0.83)(21)$ \\
Femoral neck BMD $\left(\mathrm{g} / \mathrm{cm}^{2}\right)$ & $0.66(0.08)(22)$ & $0.66(0.13)(19)$ \\
Trochanter BMD $\left(\mathrm{g} / \mathrm{cm}^{2}\right)$ & $0.59(0.0)(22)$ & $0.60(0.15)(19)$ \\
Serum alkaline phosphatase $(\mathrm{IU} / \mathrm{l})$ & $76.5(23.7)(25)$ & $75.6(25.6)(23)$ \\
Urinary calcium/creatinine $(\mathrm{mmol} / \mathrm{mmol})$ & $0.44(0.28)(25)$ & $0.64(0.89)(21)$ \\
Urinary hydroxyproline $\left./ \mathrm{creatinine}^{2} \mathrm{mg} / \mathrm{mmol}\right)$ & $2.1(1.0)(24)$ & $1.8(0.7)(20)$ \\
Urinary pyridinoline $/ \mathrm{creatinine}(\mathrm{nmol} / \mathrm{mmol})$ & $41.9(20.6)(25)$ & $48.9(28.9)(19)$ \\
Urinary deoxypyridinoline/creatinine $(\mathrm{nmol} / \mathrm{mmol})$ & $12.4(6.6)(25)$ & $10.5(6.0)(19)$ \\
& &
\end{tabular}

$\mathrm{BMD}=$ bone mineral density

${ }^{\text {aPrednisolone equivalent dose. }}$

${ }^{\mathrm{b}}$ Number of patients with available data.

mean daily corticosteroid dose was calculated as the cumulative intake of oral steroids prescribed for a patient's primary disease condition between the first intake of study medication and his/her week 104 visit, divided by the corresponding number of days.

Secondary efficacy measurements were percentage change from baseline in BMD of the lumbar spine at weeks 26,52 , and 78 . In addition, percentage change from baseline in BMD of the femoral neck, BMD of the trochanter, and serum and urine markers of bone metabolism were also compared at each time point. ANCOVA was performed on the week 52 data to complement the primary analysis, with one way ANOVA used at intermediate time points for exploratory purposes.

All analyses were performed for the intent to treat population. This population included all patients randomised into the study who received at least one dose of study drug.

\section{Results}

PATIENT SCREENING DETAILS

Of the 136 patients screened, 48 were men (mean (SD) age $64.8(10.7)$ years) and 88 were women, of which 71 were postmenopausal (mean (SD) age 66.8 (9.5) years) and 17 premenopausal (mean (SD) age 44.8 (8.9) years). Of the disease categories described, 45 patients (15 men) suffered from polymyalgia rheumatica, 52 patients (22 men) suffered from asthma, 30 patients (nine men) from systemic connective tissue disorders including systemic lupus erythematosis, Wegener's granulomatosis and polyarteritis nodosa, and five patients (two men) had skin and haematological disorders. In four patients the diagnosis was uncertain.

No patient had significant renal impairment or was receiving steroids for renal disease. This was reflected in normal PTH levels in all patients at baseline. Standard biochemical tests including vitamin $\mathrm{D}$ were similar between groups and did not differ from the normal reference range determined by the laboratory. Using the World Health Organisation criteria for definition of osteoporosis in postmenopausal women of a decrease in BMD at the spine or femoral neck of more than 2.5 standard deviations compared with young sex matched adults, ${ }^{13} 30.8 \%$ of patients did not have osteoporosis in either the spine or the hip, 8.3\% had osteoporosis of the spine alone, $21.7 \%$ the hip alone, and $39.2 \%$ the spine and hip together. The overall prevalence of osteoporosis in the screened population in the spine was therefore $47.5 \%$ and in the hip $60.9 \%$. When examined by diagnostic group the prevalence of osteoporosis at any site was $81.6 \%$ in patients with asthma, $76.7 \%$ in those with collagen vascular disease, and $48.6 \%$ in those with polymyalgia rheumatica.

\section{PATIENT DISPOSITION}

Of the 136 patients screened, 49 were found to meet the inclusion/exclusion criteria as described in the methods section and were entered into the study; 26 received cyclical etidronate (of whom 10 were men) and 23 received placebo (of whom nine were men). All patients were ambulatory. The average age of the patients at baseline was 59 , and in the postmenopausal women the years since a natural menopause was 16 . The mean (SD) corticosteroid dose at baseline was $8(4) \mathrm{mg} /$ day in the etidronate group and 7 (4) $\mathrm{mg} /$ day in the placebo group. Over the duration of the study the mean (SE) daily corticosteroid dosage (prednisolone equivalent) was $8.22(0.86)$ $\mathrm{mg} /$ day in the etidronate group and $6.38(0.86)$ $\mathrm{mg} /$ day in the placebo group. Precise dates of when corticosteroids were initiated are not available but estimated mean exposure prior to the study was approximately 8.5 years, ranging from six months to 35 years. Prestudy mean daily calcium intake ranged was $200-1700 \mathrm{mg} /$ day in the etidronate group and $200-1000 \mathrm{mg} /$ day in the placebo group.

The treatment groups were comparable with respect to all demographic and baseline data (table 1). The most frequently reported corticosteroid-requiring disease was asthma, accounting for $43 \%$ of the population (table 2 ). Each group had a representative spread of corticosteroid-requiring diseases. However, some diseases were not represented in both treatment groups. There were eight drop outs, four on etidronate and four on placebo. Results were calculated only from studies completed at the appropriate time points according to the study protocol. In the etidronate group three patients withdrew because of adverse events (myocardial infarction, death due to respiratory failure, and death due to adenocarcinoma of the lung) and one for administrative reasons. In the placebo group one patient withdrew

Table 2 Frequency of primary disease requiring corticosteroid treatment

\begin{tabular}{lll}
\hline & $\begin{array}{l}\text { Etidronate } \\
(n=26)\end{array}$ & $\begin{array}{l}\text { Placebo } \\
(n=23)\end{array}$ \\
\hline Asthma & 9 & 12 \\
Polymyalgia rheumatica & 6 & 2 \\
Systemic lupus erythematosus & 4 & 5 \\
Emphysema & 1 & 1 \\
Fasciitis & 1 & 0 \\
Giant cell arteritis & 2 & 1 \\
Polyarteritis nodosa & 1 & 1 \\
Bronchiectasis & 1 & 0 \\
Fibrosing alveolitis & 0 & 1 \\
Scleroderma & 1 & 0 \\
\hline
\end{tabular}


Table 3 Mean (SE) lumbar spine bone mineral density (percentage change from baseline)

\begin{tabular}{lll}
\hline & Etidronate $(n=26)$ & Placebo $(n=23)$ \\
\hline Week 26 & $4.33(0.85)^{\#}(22)^{\mathrm{a}}$ & $0.57(0.67)(19)^{\mathrm{a}}$ \\
Week 52 & $5.02(1.20)^{\# \star}(21)$ & $0.47(0.78)(18)$ \\
Week 78 & $5.09(0.91)^{\#}(21)$ & $0.39(1.10)(18)$ \\
Week 104 & $5.12(1.02)^{\# \star}(21)$ & $0.98(1.47)(16)$ \\
\hline
\end{tabular}

${ }^{a}$ Number of patients with available data

"Significantly different from baseline, within group analysis.

* Significantly different from placebo (analysis at weeks 52 and 104 only).

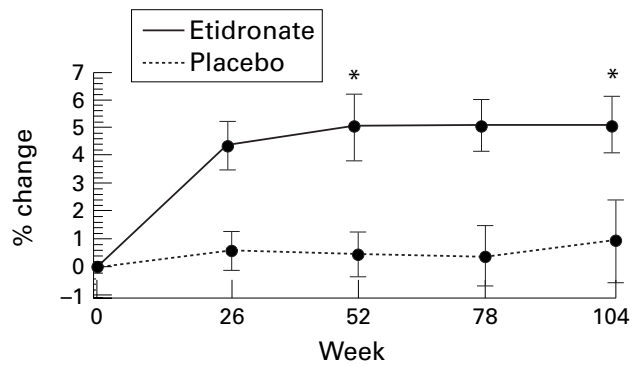

Figure 1 Mean (SE) percentage change from baseline in lumbar spine bone mineral density; *statistically significant difference between the two groups.

because of an adverse event (death due to perforated bowel) and three others for administrative reasons.

CHANGES IN BONE MINERAL DENSITY

Lumbar spine

There were no clinically relevant changes from baseline detected in the placebo group at any time during the study. However, six months of treatment with etidronate led to a mean increase from baseline of over 4\% (table 3, fig 1). Mean percentage change from baseline was maintained at each subsequent visit throughout the two year study period. The primary efficacy analysis (ANCOVA) revealed that there was a significant difference between the treatments for mean percentage change from baseline at week 104 ( $\mathrm{p}=0.007)$. The estimated mean (SE) treatment difference between etidronate and placebo was 4.50 (1.65) \% with a $95 \%$ confidence interval (CI) of $1.12 \%$ to $7.87 \%$. Patient numbers by underlying disease were small but positive treatment effects were observed across disease groups at week 104 where sufficient data were available.

The difference in mean percentage change from baseline between the treatments was also significant at week $52(\mathrm{p}=0.024)$. The estimated mean (SE) treatment difference at this time point was $4.37(1.53) \%$ with a $95 \%$ CI of $1.26 \%$ to $7.47 \%$. At each time point there was a significant difference from baseline in mean lumbar spine BMD in the etidronate group but there was no significant change from baseline in the placebo group at any time during the study.

Hip

Analysis of the hip at the femoral neck did not reveal any clinically or statistically significant treatment differences at any time, nor were any trends apparent (table 4). However, a significant increase from baseline was observed in the placebo group at week 104. In this case both placebo and treated groups showed a parallel increase between weeks 78 and 104 without the etidronate group differing significantly from baseline. Analysis of longitudinal quality control data revealed no technical explanations for this. No clinically or statistically significant treatment differences were observed at the trochanter, and the Ward's triangle was not analysed due to the large degree of variability observed at this site.

\section{Bone markers}

Mean serum alkaline phosphatase remained approximately at baseline levels for the placebo group whilst a decrease in mean serum alkaline phosphatase was observed in the etidronate group, but this was not statistically significant. The estimated difference in mean percentage change from baseline was $-12.32 \%$ (95\% CI -23.84 to -0.80$)$ at week $26,-13.20 \%,(95 \%$ CI -27.68 to 1.29 ) at week 52 , and $-9.87 \%$ (95\% CI -26.31 to 6.57$)$ at week 104 .

Urinary hydroxyproline/creatinine ratios were suppressed in the etidronate group with a significant difference in mean percentage change from baseline between treatment groups at week 26 . The estimated mean treatment difference was $-41.27 \%$ (95\% CI -77.35 to -5.70$)$. No significant differences between groups were observed thereafter. Analysis of pyridinoline/creatinine showed a similar profile for both treatment groups throughout the study. There was no significant difference in mean percentage change from baseline between the treatment groups at any time during the study. Deoxypyridinoline/creatinine ratios were increased in the placebo group compared with baseline throughout the study but little change was observed in the etidronate treated patients. There were, however, no significant treatment differences in mean percentage change from baseline at any time during the study.

\section{SAFETY DATA}

Thirty six patients $(80 \%)$ reported one or more adverse events which were evenly distributed amongst the treatment groups; $17 / 26(65 \%)$ in the etidronate group and $19 / 23(83 \%)$ in the placebo group. The most common adverse events in the study as a whole were respiratory infections (experienced by five patients in the etidronate group and two in the placebo group), back pain (four in the etidronate group and none in the placebo group), and accidental injury (four in the placebo group alone). Most of the adverse events were mild or moderate in severity and resolved before the end of the study.

Table 4 Mean (SE) femoral neck bone mineral density (percentage change from baseline)

\begin{tabular}{lrl}
\hline & Etidronate $(n=26)$ & Placebo $(n=23)$ \\
\hline Week 26 & $1.09(0.91)(19)^{\mathrm{a}}$ & $1.43(1.26)(18)^{\mathrm{a}}$ \\
Week 52 & $-0.08(0.67)(16)$ & $1.57(1.27)(16)$ \\
Week 78 & $0.29(1.16)(18)$ & $1.29(1.53)(16)$ \\
Week 104 & $2.47(1.29)(18)$ & $3.64(1.67)^{\#}(15)$ \\
\hline
\end{tabular}

${ }^{a}$ Number of patients with available data.

"Significantly different from baseline, within group analysis. 
Two patients in the placebo group reported moderate to severe upper gastrointestinal adverse events but no events of this nature were reported in the etidronate group. Eight (35\%) patients in the placebo group and five $(19 \%)$ in the etidronate group had serious adverse events but none were considered to be related to study treatment. Two deaths were reported during the study period, one in each treatment group, neither of which were considered to be related to the study treatment, and one death occurred after the study was completed. New vertebral fractures were detected in two patients in the etidronate group and in one in the placebo group during the course of the study. There were no clinically significant laboratory abnormalities or changes in vital signs or physical examination that were attributed to treatment.

\section{Discussion}

This study shows that, in patients who have already experienced bone loss, treatment with an intermittent cyclical regimen of etidronate and calcium/vitamin $\mathrm{D}$ supplementation is effective not only in preventing any further bone loss in the lumbar spine but in increasing bone mass from baseline levels. The initial selection of patients ensured that the study focused on those who had been exposed to relatively high doses of corticosteroids for periods of more than six months and who had a lumbar spine bone density (mean L2-L4 vertebrae) of one standard deviation or more below the age and sex matched mean for the normal population. After only six months of treatment with etidronate the mean bone mass at the lumbar spine was significantly increased from baseline by $4.3 \%$ which then plateaued over the remaining course of the study. Interestingly, the placebo patients remained at baseline levels throughout. This may suggest that, since these patients had already been exposed to corticosteroids for some time before enrolment into the study, the effects of the corticosteroids on bone loss may have ceased. However, all patients took calcium and vitamin D supplementation which may have contributed to the maintenance of bone mass in the placebo patients, as one would expect some degree of age related bone loss. Since recent data suggest that changes in bone mass may underestimate the overall effects on fracture reduction in postmenopausal osteoporosis, ${ }^{14}$ it may be reasonable to propose that the increases in bone mass observed in the etidronate treated patients may confer a benefit in reducing the subsequent risk of vertebral fractures. Clearly, much larger controlled studies would be needed to confirm this.

No clinically or statistically significant treatment effects were observed at any site of the hip. This was also the case in the one year study by Adachi et $a l^{15}$ but differs from that of Struys et $a l^{16}$ in which 12 months of treatment with cyclical etidronate resulted in significant mean increases from baseline in both the lumbar spine and proximal femur of $5.7 \%$ and $6.8 \%$, respectively. Some evidence suggests that corticosteroids may have different effects on different skeletal regions depending upon the composition of that region - that is, predominance of trabecular bone in the axial skeleton (for example, the lumbar spine) and cortical bone in the appendicular skeleton (for example, the hip)..$^{217}$ The study by Sambrook et $a l,{ }^{5}$ however, suggests that bone losses due to corticosteroids at these sites are comparable. The screening phase of 136 patients in this study showed an overall prevalence of hip osteoporosis of $60.9 \%$ and spine osteoporosis of $47.5 \%$, lending further support to this view.

Although a broad range of disease types were included in the study, most of the patients $(43 \%)$ suffered from asthma. Patients with rheumatoid arthritis and inflammatory bowel disease were excluded from the study. The numbers of patients in other disease groups were too small to enable meaningful analysis of treatment effects by disease category, but in general the difference between treatment groups within each disease category was positive in favour of etidronate for lumbar spine BMD. This suggests that the effects of etidronate therapy in the prevention of bone loss in corticosteroid induced bone disease is independent of the underlying disease. The difference in the effects of treatment between men and women was not determined as the patient numbers did not allow for meaningful analysis.

The analysis of serum total alkaline phosphatase showed, as expected, a suppression of levels in the etidronate treated patients. These differences were not statistically significant, however. Similarly, no significant group differences were observed for urinary bone turnover markers.

In conclusion, the results of this study demonstrate that intermittent cyclical treatment with etidronate and calcium with vitamin $\mathrm{D}$ supplementation significantly increases bone density in the lumbar spine in patients with osteoporosis resulting from long term treatment with corticosteroids. The treatment was well tolerated with an adverse event profile comparable to placebo. This would therefore appear to be a suitable intervention for patients who have already experienced bone loss due to corticosteroid therapy.

The authors would like to acknowledge the contribution of the following for the assistance in the study: B Bloom, C Mackintosh, H Berry, J Costello, B Bourke, C Goodwill, G Hughes, D Hutchinson, A Jariwalla, J Moxham, I O'Brien, E Sawicka, and P Williams. This study was supported by Procter \& Gamble Pharmaceuticals, European Research \& Development.

1 Lukert BP, Raisz LG. Glucocorticoid-induced osteoporosis: Lukert BP, Raisz LG. Glucocorticoid-induced osteoporosis: pathogenesis and

2 Adinoff AD, Hollister JR. Steroid induced fractures and bone loss in patients with asthma. N Engl F Med 1983;309: 265-8.

3 Dempster DW. Bone histomorphometry in glucocorticoidinduced osteoporosis. F Bone Miner Res 1989;4:137-41.

4 Lukert BP, Raisz LG. Glucocorticoid-induced osteoporosis. Rheum Dis Clin North Am 1994;20:629-50.

5 Sambrook P, Birmingham J, et al. Prevention of corticosteroid osteoporosis. A comparison of calcium, calcitriol, and calcitonin. N Engl F Med 1993;328:1747-52.

6 Reid IR, King AR, Alexander CJ, et al. Prevention of steroid-induced osteoporosis with (3-aminohydroxypropylidene)-1,1-bisphosphonate (APD). Lancet 1988;i:143-6.

7 Sebaldt RJ, Adachi JD, Bensen WG, et al. Intermittent cyclic therapy with etidronate prevents corticosteroid-induced bone loss: two years of follow-up. Scand $\mathcal{F}$ Rheumatol 1996; 25(Suppl 103): $91-3$. 
8 Pitt P, Compston JE, Trevidi P, et al. Systemic bone changes accompanying early rheumatoid arthritis in patients treated only with non-steroidal anti-inflammatory drugs. Clin Orthop Rel Res 1994;298:250-8.

9 Compston JE, Judd D, Crawley EO, et al. Osteoporosis in patients with inflammatory bowel disease. Gut 1987;28: $410-5$.

10 Suleiman S. A screening questionnaire for post-menopausal osteopenia. $1995 \mathrm{PhD}$ Thesis, University of London.

11 Li F, Pitt P, Sherwood R, et al. Biochemical markers of bone turnover in women with surgically treated carcinoma of the breast. Eur f Clin Invest 1993;23:566-71.

12 Genant HK, Wu CY, Van Kuijk C, et al. Vertebral fracture assessment using a semiquantitative technique. 7 Bone Miner Res 1993;8:1137-48.

13 Kanis JA. Assessment of fracture risk and its application to the screening for postmenopausal osteoporosis: synopsis of

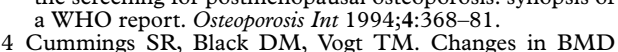
substantially underestimate the anti-fracture effects of alendronate and other antiresorptive drugs. $\mathcal{F}$ Bone Miner Res 1996;11(Suppl 1):Abstract 29.

15 Adachi JD, Cranney A, Goldsmith CH, et al. Intermittent cyclic therapy with etidronate in the prevention of corticosteroid induced bone loss. F Rheumatol 1994;21:1922-6.

16 Struys A, Snelder AA, Mulder H. Cyclical etidronate reverses bone loss of the spine and proximal femur in patients with established corticosteroid-induced osteoporosis. Am f Med 1995;99:235-42.

17 Schaadt O, Bohr H. Bone mineral in lumbar spine, femoral neck and femoral shaft measured by dual photon absorptiometry with 153-gadolineum in prednisone treatment. $A d v$ Exp Med Biol 1984;171:201-8. 